Available online at www.eccomasproceedia.org

Eccomas Proceedia COMPDYN (2021) 1934-1940

ECCOMAS

Proceedia
COMPDYN 2021

$8^{\text {th }}$ ECCOMAS Thematic Conference on Computational Methods in Structural Dynamics and Earthquake Engineering M. Papadrakakis, M. Fragiadakis (eds.) Streamed from Athens, Greece, 28 - 30 June 2021

\title{
AN EFFICIENT PRECONDITIONER FOR MODAL FREQUENCY RESPONSE
}

\author{
T. Willerding \\ INTES GmbH \\ Breitwiesenstraße 28 \\ 70565 Stuttgart, Germany \\ e-mail: tobias.willerding@intes.de
}

\begin{abstract}
In frequency response analysis, it is of interest to simulate the dynamic response of a system for many excitation frequencies. In order to accelerate the calculation, the system is first transformed to modal space by calculating a limited set of eigenvectors and eigenvalues. In this way, the number of degrees of freedom can be reduced from e.g. 50 million to 50,000. However, due to the existence of structural and viscous damping, the system matrix in modal space is dense in a general case.

In this work, a method to generate a preconditioner for the solution of modal frequency response is proposed. First, a decomposition of the system matrix at one excitation frequency is performed using a direct method (e. g. LU decomposition). In a second step, this decomposition is modified for different excitation frequencies in an efficient way, by using a complex eigenvalue problem. Although the modified decomposition is no longer exact, it has excellent characteristics to be used as a preconditioner in an iterative method. Using a complex eigenvalue problem and a decomposition update has already been proposed in [1] for a direct solution method. However, in this work, the focus is on an iterative method.

By making use of the structure of the system matrix, it is possible to split the product $\mathbf{A x}$ in the iterative solver in multiple parts to iterate many right hand sides (for equal or different excitation frequencies) at once. In this way, the iterative solver can make use of dense matrixmatrix multiplications, using BLAS Level-3 [6], which results in better performance.
\end{abstract}

Keywords: Preconditioner, modal frequency response, iterative solver, complex eigenvalue problem

ISSN:2623-3347 @ 2021 The Authors. Published by Eccomas Proceedia.

Peer-review under responsibility of the organizing committee of COMPDYN 2021.

doi:10.7712/120121.8612.18811 


\section{INTRODUCTION}

In modern finite element software (e.g. PERMAS [9]), an important problem is the calculation of the response of a system subject to harmonic loading. The system can represent a large finite element model such as a car (see figure 1), an aircraft or a ship. It is also important in the design of machine tools [3], spaceflight [4] or in acoustic analysis [5]. The dynamic response is calculated with frequency response analysis. This analysis is performed for many (e.g. 1000 or more) excitation frequencies. The solution at each excitation frequency requires the solution of a linear equation system with millions of unknowns in modern applications. In order to speed up the calculation, the dynamic system is typically transformed to modal space. This is achieved by calculating a limited number of eigenvalues and eigenvectors of the original system. The quality of the transformation depends on the dimension of the modal space, e.g. the number of calcuated eigenvalues. !

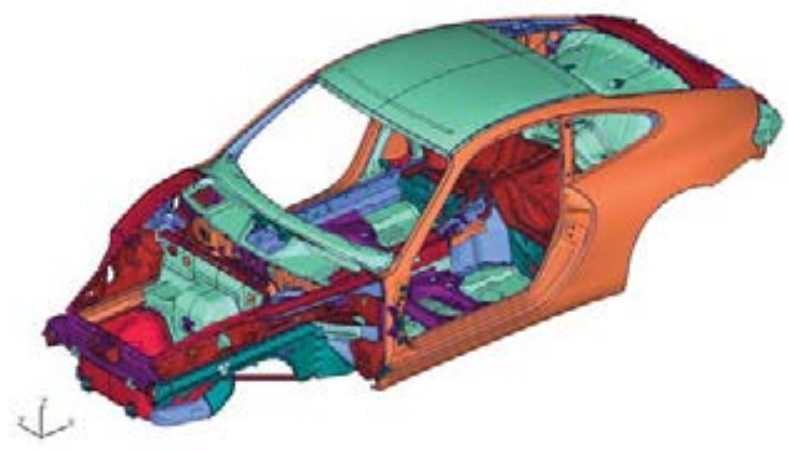

Figure 1: Porsche 996, by courtesy of Dr. Ing. h.c. F. Porsche AG

In modal space, the mass and stiffness matrix are diagonal matrices, which is very convenient for the frequency response analysis. However, a finite element model also often contains damper elements or structural damping, which results in dense damping matrices in modal space. Kim and Benninghof [1] noted, one of the two dense matrices can be diagonalized in modal space by solving another complex eigenvalue problem. However, one dense matrix remains. If this matrix is of low rank, the inverse can be updated easily with the Woodbury matrix identity as noted by Kim and Bennighof. However, if the viscous damping matrix has full rank, there is no advantage in doing this.

In this work, an idea is presented to solve this problem with an iterative solver, where the inverse of the system matrix at a reference frequency is calculated and this solution is modified by solving a complex eigenvalue problem, similiar to the work by Kim and Bennighof. The iterative solver could be a purely iterative method (e.g. Jacobi iteration) or a more sophisticated method like GMRES [2].

\section{THEORY}

In frequency response, the solutions $\tilde{\mathbf{x}} \in \mathbb{C}^{n}$ to the equation

$$
\left(-\omega^{2} \tilde{\mathbf{M}}+i \omega \tilde{\mathbf{D}}+i \tilde{\mathbf{H}}+\tilde{\mathbf{K}}\right) \tilde{\mathbf{x}}=\tilde{\mathbf{A}}(\omega) \tilde{\mathbf{x}}=\tilde{\mathbf{b}}
$$

for different $\omega \in \mathbb{R}$ have to be calculated. $\tilde{\mathbf{M}} \in \mathbb{R}^{n \times n}$ is the mass matrix, $\tilde{\mathbf{D}} \in \mathbb{R}^{n \times n}$ is the viscous damping matrix, $\tilde{\mathbf{H}} \in \mathbb{R}^{n \times n}$ is the structural damping matrix and $\tilde{\mathbf{K}} \in \mathbb{R}^{n \times n}$ is 
the stiffness matrix. In addition, on the right hand side, there is a loading vector $\tilde{\mathbf{b}} \in \mathbb{C}^{n}$. $i$ represents the imaginary unit. The matrices $\tilde{\mathbf{M}}, \tilde{\mathbf{K}}, \tilde{\mathbf{D}}$ and $\tilde{\mathbf{H}}$ are symmetric in most cases, but they may also include rotational effects or special elements, that result in a non-symmetric problem. In this work, only the symmetric case is considered. Furthermore, it is assumed that the matrices $\tilde{\mathbf{D}}$ and $\tilde{\mathbf{H}}$ are positive semi-definite.

In modal frequency response, a generalized eigenvalue problem for $\tilde{\mathbf{K}}$ and $\tilde{\mathbf{M}}$ is solved for a limited number of eigenvalues $m$ and the system is transformed to modal space. Using a transformation matrix $\Phi$, we can transform the matrices to modal space: $\mathbf{M}=\Phi^{\mathrm{T}} \tilde{\mathbf{M}} \boldsymbol{\Phi}$, $\mathbf{D}=\boldsymbol{\Phi}^{\mathrm{T}} \tilde{\mathbf{D}} \boldsymbol{\Phi}, \mathbf{H}=\boldsymbol{\Phi}^{\mathrm{T}} \tilde{\mathbf{H}} \boldsymbol{\Phi}, \mathbf{K}=\boldsymbol{\Phi}^{\mathrm{T}} \tilde{\mathbf{K}} \boldsymbol{\Phi}$. The load is transformed as $\mathbf{b}=\boldsymbol{\Phi}^{\mathrm{T}} \tilde{\mathbf{b}}$ and the displacement vector is $\mathbf{x}=\Phi^{\mathrm{T}} \tilde{\mathbf{x}}$. Finally we arrive at the following equation system in modal space:

$$
\left(-\omega^{2} \mathbf{M}+i \omega \mathbf{D}+i \mathbf{H}+\mathbf{K}\right) \mathbf{x}=\mathbf{A}(\omega) \mathbf{x}=\mathbf{b} .
$$

The transformed system has a diagonal mass matrix $\mathbf{M}$, a diagonal stiffness matrix $\mathbf{K}$ and a dense viscous damping matrix $\mathbf{D}$ and a dense structural damping matrix $\mathbf{H}$. The system size is reduced from $n \times n$ to $m \times m$. In this work, this system is investigated to be solved with an iterative method. In general, iterative methods need a preconditioner to converge within an acceptable number of iterations.

\section{PRECONDITIONING}

In order to judge the quality of the preconditioner, the convergence criterion of a purely iterative solver is used, because it is very simple and easy to understand. The equation $\mathbf{A x}=\mathbf{b}$ is iteratively solved using a preconditioner $\mathbf{P}$. This means that every eigenvalue $\lambda$ of $\mathbf{I}-\mathbf{P}^{-1} \mathbf{A}$ must fulfill

$$
\left|\lambda\left(\mathbf{I}-\mathbf{P}^{-1} \mathbf{A}\right)\right|<1 .
$$

It is desirable to construct a preconditioner that works for $k$ values $\omega_{1}<\omega_{i}<\omega_{k}$. First, a reference circular frequency $\omega_{\text {ref }}$ with $\omega_{1}<\omega_{\text {ref }}<\omega_{k}$ is selected and a direct solution method (e.g. LU decomposition) is used to calculate the inverse of $\mathbf{A}_{\text {ref }}=\mathbf{A}\left(\omega_{\text {ref }}\right)$. However, this reference inverse is insufficient as a general preconditioner and it must be modified to adapt it to each individual circular frequency $\omega_{i}$ as will be seen later.

The idea consists of approximating the difference between $\mathbf{A}(\omega)$ and $\mathbf{A}\left(\omega_{\text {ref }}\right)$ with a matrix $\mathbf{S}$ and an $\omega$-dependent scalar factor $f(\omega)$. This approximation of $\mathbf{A}(\omega) \approx \mathbf{P}=\mathbf{A}\left(\omega_{\text {ref }}\right)+$ $f(\omega) \mathbf{S}$ is taken as preconditioner.

In general, the difference between $\mathbf{A}(\omega)$ and $\mathbf{A}\left(\omega_{\text {ref }}\right)$ can be expressed as

$$
\begin{aligned}
\mathbf{A}(\omega) & =\mathbf{A}\left(\omega_{\text {ref }}\right)+\left(\left(\mathbf{A}(\omega)-\mathbf{A}\left(\omega_{\text {ref }}\right)\right)\right. \\
& =\mathbf{A}\left(\omega_{\text {ref }}\right)+\left(-\omega^{2}+\omega_{\text {ref }}^{2}\right) \mathbf{M}+i\left(\omega-\omega_{\text {ref }}\right) \mathbf{D}
\end{aligned}
$$

In this work, only an approximation of the mass matrix is considered. The reference matrix $\mathbf{A}_{\text {ref }}$ is modified with the mass matrix $\mathbf{M}$, multiplied with a scalar function $f(\omega)$.

$$
\mathbf{A}(\omega) \approx \mathbf{A}\left(\omega_{\text {ref }}\right)+\left(-\omega^{2}+\omega_{\text {ref }}^{2}\right) \mathbf{M}=\mathbf{A}\left(\omega_{\text {ref }}\right)+f(\omega) \mathbf{M}=\mathbf{P}
$$

The expression is now transformed as follows,

$$
\begin{aligned}
\mathbf{P}^{-1}=\left(\mathbf{A}_{\text {ref }}+f(\omega) \mathbf{M}\right)^{-1} & =\left(\mathbf{A}_{\text {ref }}\left(\mathbf{I}+\mathbf{A}_{\text {ref }}^{-1} \mathbf{M} f(\omega)\right)\right)^{-1} \\
& =\left(\mathbf{I}+\mathbf{A}_{\text {ref }}^{-1} \mathbf{M} f(\omega)\right)^{-1} \mathbf{A}_{\text {ref }}^{-1}
\end{aligned}
$$


Next, we solve a complex eigenvalue problem of $\mathrm{A}_{\text {ref }}^{-1} \mathrm{M}=\mathrm{Q} \Lambda \mathrm{Q}^{-1}$. We can now replace $\mathbf{A}_{\mathrm{ref}}^{-1} \mathbf{M}$ with an orthonormal eigenvector matrix $\mathrm{Q}$ and the eigenvalue diagonal matrix $\Lambda$ :

$$
\begin{aligned}
\mathbf{P}^{-1}=\left(\mathbf{A}_{\text {ref }}+f(\omega) \mathbf{M}\right)^{-1} & =\left(\mathbf{Q Q} \mathbf{Q}^{-1}+f(\omega) \mathbf{Q} \mathbf{\Lambda} \mathbf{Q}^{-1}\right)^{-1} \mathbf{A}_{\text {ref }}^{-1} \\
& =\mathbf{Q}(\mathbf{I}+f(\omega) \boldsymbol{\Lambda})^{-1} \mathbf{Q}^{-1} \mathbf{A}_{\text {ref }}^{-1} .
\end{aligned}
$$

This expression for $\mathbf{P}^{-1}$ is quite interesting, because first the right hand side is multiplied with the reference inverse $\mathbf{A}\left(\omega_{\text {ref }}\right)^{-1}$ and then with the eigenvector matrices $\mathbf{Q}$ and a diagonal matrix, that is different for each $\omega$. With the definitions $\mathbf{U}=\mathbf{Q}, \mathbf{V}=\mathrm{Q}^{-1} \mathbf{A}_{\text {ref }}^{-1}$ and $\mathbf{G}(\omega)=(\mathbf{I}+f(\omega) \boldsymbol{\Lambda})^{-1}$, we can write this as

$$
\mathbf{P}^{-1}=\left(\mathbf{A}_{\text {ref }}+f(\omega) \mathbf{M}\right)^{-1}=\mathbf{U G}(\omega) \mathbf{V} .
$$

As can be seen, the only term that is $\omega$-dependent is $\mathbf{G}$, which is a diagonal matrix. In the next section, it will be shown that this preconditioner can be evaluated in a very efficient manner.

However, first, the preconditioner quality is discussed. GNU Octave [7] is used for the evaluation of the eigenvalues. A matrix A of the size $m=100$ is selected, which corresponds to 100 modes calculated. The stiffness matrix $\mathbf{K}$ is the identity matrix in modal space. The mass matrix $\mathbf{M}$ is also diagonal and we set the valus of $\mathbf{M}$ equal to $M_{i i}=1 / i^{2}$, which corresponds to circular eigenfrequencies $\omega_{i}=i$. Furthermore symmetric, positiv semi-definite matrices are chosen for $\mathbf{H}$ and $\mathbf{D}$. The eigenvalues of $\mathbf{H}$ and $\mathbf{D}$ are set to be randomly equally distributed between 0 and $d_{\max }$ or $h_{\max }$, respectively. In addition, we select a reference frequency $\omega=50$ and look at the absolute largest eigenvalue between $\omega=0$ and $\omega=100$. Depending on the seed of the random generator, the eigenvalues of $\mathbf{I}-\mathbf{P}^{-1}(\omega) \mathbf{A}(\omega)$ look slightly different, but the general picture is as seen in figure 2 and 3.

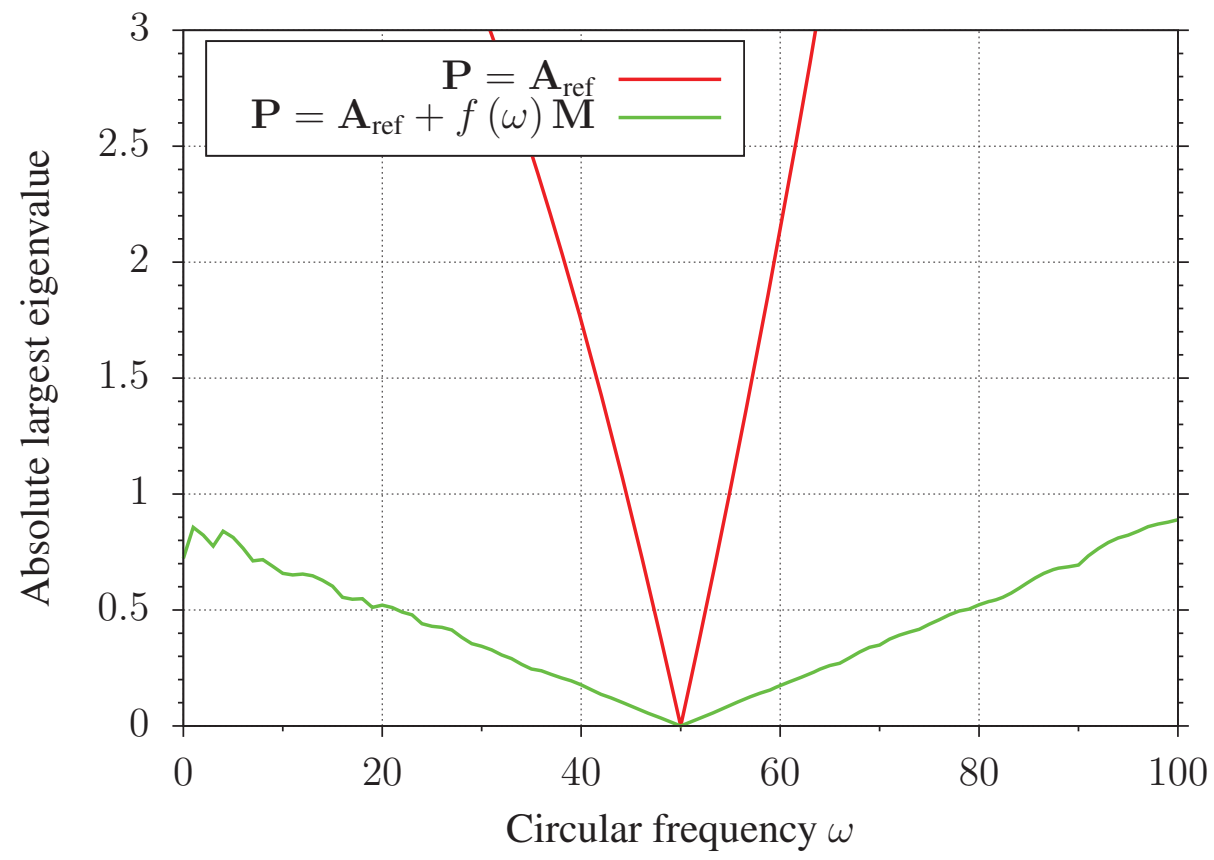

Figure 2: Plot of the absolute largest eigenvalue of $\mathbf{I}-\mathbf{P}^{-1}(\omega) \mathbf{A}(\omega)$ for two different preconditioners, inverse only and inverse with mass matrix modification. $d_{\max }=1.0 \times 10^{-4}$ 


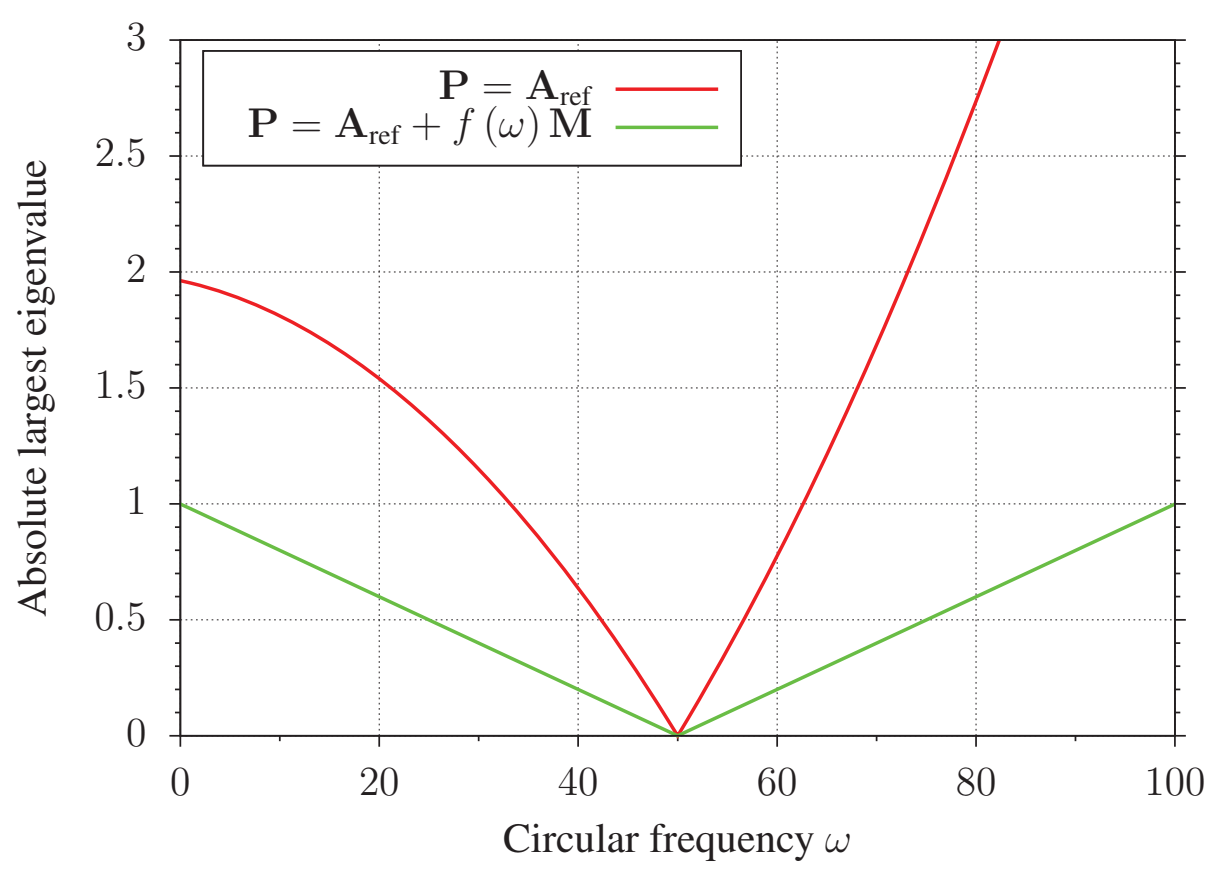

Figure 3: Plot of the absolute largest eigenvalue of $\mathbf{I}-\mathbf{P}^{-1}(\omega) \mathbf{A}(\omega)$ for two different preconditioners, inverse only and inverse with mass matrix modification. $d_{\max }=1.0 \times 10^{-2}$

Using these definitions for $\mathbf{M}, \mathbf{D}, \mathbf{H}$ and $\mathbf{K}$, figures 2 and 3 show the absolute largest eigenvalue of $\mathbf{I}-\mathbf{P}^{-1}(\omega) \mathbf{A}(\omega)$ for different values of $d_{\max }$. As can be seen in the figure, at $\omega=\omega_{\text {ref }}$, the absolute largest eigenvalue is zero as expected. The inverse is - of course - the perfect preconditioner. For other frequencies the absolute largest eigenvalue is monotonously rising with $\omega$. If only the inverse at the reference circular frequency $\omega_{\text {ref }}$ is taken as preconditioner, it can be seen that the absolute largest eigenvalue is rising quickly, depending on damping $d_{\max }$. The higher the damping, the better the inverse for neighbouring frequencies. If the inverse is modified with the above mentioned approach, the largest absolute eigenvalue is rising much more slowly and remains below or equal to 1 in the investigated interval. Even in cases, where the inverse-only preconditioner rises quickly as seen in figure 2 , the absolute largest eigenvalue of the modified inverse stays below 1 . The reason for this will be investigated in future work.

\section{IMPLEMENTATION}

In order to use this formulaton, it is useful to have an efficient evaluation of the product $\mathbf{A}(\omega) \mathbf{x}$ and the application of the preconditioner. The matrix is defined as

$$
\left(-\omega^{2} \mathbf{M}+i \omega \mathbf{D}+i \mathbf{H}+\mathbf{K}\right) \mathbf{x}=\mathbf{b} .
$$

In an iterative solver, the desired value is the residual $\mathbf{r}$ with

$$
\mathbf{r}=\mathbf{b}-\left(-\omega^{2} \mathbf{M}+i \omega \mathbf{D}+i \mathbf{H}+\mathbf{K}\right) \mathbf{x}
$$

This can be rearranged to

$$
\mathbf{r}=\mathbf{b}+\mathbf{M} \mathbf{x} \omega^{2}-i \mathbf{D} \mathbf{x} \omega-i \mathbf{H} \mathbf{x}-\mathbf{K} \mathbf{x}
$$


By defining the matrix

$$
\boldsymbol{\Omega}=\left[\begin{array}{cccc}
\omega_{1} & 0 & \cdots & 0 \\
0 & \omega_{2} & \ddots & 0 \\
\vdots & \ddots & \ddots & \vdots \\
0 & \cdots & 0 & \omega_{q}
\end{array}\right]
$$

and assembling $q$ right hand sides

$$
\mathbf{B}=\left[\begin{array}{llll}
\mathbf{b}\left(\omega_{1}\right) & \mathbf{b}\left(\omega_{2}\right) & \cdots & \mathbf{b}\left(\omega_{q}\right)
\end{array}\right],
$$

it is possible to calculate residuals for multiple right hand sides with different $\omega_{i}$ at once instead of calculating each vector $\mathbf{x}$ with a different $\mathbf{A}(\omega)$. With the iteration vectors

$$
\mathbf{X}=\left[\begin{array}{llll}
\mathbf{x}\left(\omega_{1}\right) & \mathbf{x}\left(\omega_{2}\right) & \cdots & \mathbf{x}\left(\omega_{q}\right)
\end{array}\right]
$$

the $\mathbf{R}$ can be expressed as

$$
\mathbf{R}=\mathbf{B}+\mathbf{M X} \Omega^{2}-i \mathbf{D X} \Omega-i \mathbf{H X}-\mathbf{K X} .
$$

The preconditioner is applied to the residual $\mathbf{r}$ in order to get an increment on $\mathbf{x}$ :

$$
\mathrm{d} \mathbf{x}=\mathbf{P}^{-1} \mathbf{r}=\mathbf{U G}(\omega) \mathbf{V r} .
$$

The diagonal matrix $\mathbf{G}(\omega)$ can be converted to a column vector $\mathrm{g}$. Next, preconditioner vectors for multiple right hand sides can be assembled to form a matrix

$$
\mathbf{G}_{R}=\left[\begin{array}{llll}
\mathbf{g}\left(\omega_{1}\right) & \mathbf{g}\left(\omega_{2}\right) & \cdots & \mathbf{g}\left(\omega_{q}\right) \cdot
\end{array}\right]
$$

In other words, just like the product $\mathbf{A}(\omega) \mathbf{x}$, also in this case multiple vectors can be iterated at once:

$$
\mathrm{d} \mathbf{X}=\mathbf{P}^{-1} \mathbf{R}=\mathbf{U}\left(\mathbf{G}_{R} \odot(\mathbf{V R})\right) .
$$

Here $\odot$ symbolizes the Hadamard product [8]. The Hadamard product is a entry-wise multiplication of matrices. Both matrices must have the same dimension. The total operation count per iteration and vector is a bit higher than compared to the case, where each vector is iterated with the assembled matrix. However, modern CPU architecture is limited by memory bandwidth and the proposed method can make full use of the CPU as only dense matrix multiplication are performed. These can be efficiently performed with Level-3 BLAS [6], for example. A benchmark test case will be performed in future work.

\section{CONCLUSIONS}

In this work, the construction of a preconditioner for modal frequency response has been presented. The inverse of the system matrix is calculated at a reference frequency with a direct solution method (e.g. LU decomposition). The decomposition is modified by first solving a complex eigenvalue problem. By solving a complex eigenvalue problem, the frequency specific modification information is limited to diagonal matrices. These diagonal matrices can be transformed to a column vector and efficiently evaluated by performing a Hadamard product with the iteration vectors. The absolute value of the eigenvalues of $\mathbf{I}-\mathbf{P}^{-1} \mathbf{A}$ are below or equal 1 , if the reference frequency is in the middle of the interval of interest. The absolute largest eigenvalue of $\mathbf{I}-\mathbf{P}^{-1} \mathbf{A}$ is slowly rising from zero at the reference frequency to 1 at the integration bounds. 


\section{REFERENCES}

[1] Chang-Wan Kim, J.K. Bennighof, Fast frequency response analysis of partially damped structures with non-proportional viscous damping, Journal of Sound and Vibration, Volume 297, Issues 35, 2006, Pages 1075-1081.

[2] Saad, Youcef and Schultz, Martin H., GMRES: A Generalized Minimal Residual Algorithm for Solving Nonsymmetric Linear Systems, SIAM Journal on Scientific and Statistical Computing, Volume 7, Number 3, Pages 856-859, 1986

[3] R. Helfrich, B. Kirchgäßner, G. Nötzel-Steidle, Optimizing the Dynamic System Behaviour of Machine Tools, NAFEMS World Congress 2019, Quebec 17-20 June 2019 (PDF).

[4] R. Helfrich, J. Marchesini, Dynamic substructuring with mixed boundary conditions to cope with complex structural assemblies, European Conference on Spacecraft Structures, Materials \& Environmental Testing, Braunschweig, Germany, 1-4 April 2014

[5] R. Helfrich, M. Spriegel, Noise propagation from vibrating structures, NAFEMS World Congress 2015, San Diego 21-24 June 2015

[6] Basic Linear Algebra Subprograms, http: / / www . netlib.org/blas /

[7] GNU Octave, https://www.gnu.org/software/octave/

[8] For more details on the Hadamard product, see https://en.wikipedia.org/ wiki/Hadamard_product_(matrices)

[9] PERMAS Users Reference Manual, INTES Publication No. 450 